\title{
INFLUÊNCIA DA CONDUTIVIDADE ELÉTRICA, CONCENTRAČ̃̃ IÔNICA E VAZÃO DE SOLUÇÕES NUTRITIVAS NA PRODUÇẪO DE ALFACE HIDROPÔNICA
}

\author{
Effect of electric conductivity, ionic concentration and flow of nutrient solutions in the \\ production of hidroponic lettuce
}

\author{
Celso Helbel Junior ${ }^{1}$, Roberto Rezende ${ }^{2}$, Paulo Sérgio Lourenço de Freitas ${ }^{3}$, \\ Antônio Carlos Andrade Gonçalves ${ }^{3}$, José Antônio Frizzone ${ }^{4}$
}

\begin{abstract}
RESUMO
Objetivou-se, neste trabalho, estudar os efeitos de diferentes soluções nutritivas aplicadas em duas vazões na produção hidropônica da alface (Lactuca sativa), cultivada pelo sistema NFT (Nutritive Film Technique). O experimento foi conduzido em casa de vegetação, na unidade de pesquisa em agricultura irrigada da Universidade Estadual de Maringá. Os tratamentos constaram de três soluções nutritivas com concentrações diferentes ( $\mathrm{S} 1=0,8 \mathrm{dS} / \mathrm{m}, \mathrm{S} 2=1,2 \mathrm{dS} / \mathrm{m}$ e S3=2,5 dS/m), combinadas com duas vazões de aplicação (V1=0,8 L. $\mathrm{min}^{-1} \mathrm{e}$ V2=1,2 L. $\mathrm{min}^{-1}$ ) nos canais de cultivo em esquema fatorial 3 x 2. Para avaliar o efeito dos tratamentos no desenvolvimento da cultura os dados foram submetidos à análise de variância (teste $\mathrm{F}$ ), e os resultados avaliados através de teste de média de Tukey $(5 \%)$. A análise dos resultados permitiu concluir que os fatores solução e vazão não apresentaram interação significativa e que os maiores valores avaliados de biomassa fresca e diâmetro do caule foram obtidos no tratamento solução $2(\mathrm{CE}=$ $1,2 \mathrm{dS} / \mathrm{m})$
\end{abstract}

Termos para indexação: Lactuca sativa , soluções nutritivas, vazões e hidroponia.

\section{ABSTRACT}

The porpose of this work was to study the effect of different nutrient solutions applied in two outflow in the hidroponic yeld of lettuce (Lactuca sativa). This research was accomplished at greenhouse through hydroponic system NFT in Maringá State University. The treatments consisted of three nutrient solutions ( $\mathrm{S} 1=0,8 \mathrm{dS} / \mathrm{m}, \mathrm{S} 2=1,2 \mathrm{dS} / \mathrm{m}$ e S3=2,5 dS/m) combined with two flows of application $\left(\mathrm{V} 1=0,8 \mathrm{~L} \cdot \mathrm{min}^{-1} \mathrm{e} \mathrm{V} 2=1,2 \mathrm{~L} \cdot \mathrm{min}^{-1}\right)$ in the growing channels of cultivation. The flows of application of the solutions were $0,8 \mathrm{~L} \cdot \mathrm{min}^{-1}(\mathrm{~V} 1)$ and 1,2 L.min ${ }^{-1}(\mathrm{~V} 2)$, placed alternately in the growing channels in a $3 \times 2$ factorial scheme. To evaluate the effect of treatments on the culture development the data were submitted to the variance analysis (Test F), and the results were evaluated through Tukey (5\%) average test. The analysis of the results allowed to conclude that the factories nutrient solution and flow did not show significant interaction. The greater values evaluated of biomass fresh and diameter of stem by plant were obtained in treatment solution $2(\mathrm{EC}=1,2 \mathrm{dS} / \mathrm{m})$.

Index terms: Lactuca sativa, nutrient solutions, flow and hydropony.

(Recebido em 5 de março de 2007 e aprovado em 12 de fevereiro de 2008)

\section{INTRODUÇÃo}

A hidroponia é uma técnica de cultivo de plantas em meio líquido que tem se expandido no mundo todo como meio de cultivo de hortaliças, pois permite o plantio durante todo ano, além de atender perfeitamente às exigências de produção com uniformidade, alta qualidade, alta produtividade, desperdício mínimo de água e nutrientes e o mínimo de uso de defensivos agrícolas (ALBERONI, 1998).
A alface (Lactuca sativa L.), por ocupar pouco espaço, atingir mais rapidamente o ponto de comercialização e pelo rápido retorno financeiro, é a cultura que vem ganhando cada vez mais espaço na produção hidropônica (ZITO et al., 1994).

Entretanto, para o sucesso do cultivo hidropônico é imprescindível conhecer os aspectos nutricionais e de manejo das plantas nesse sistema de produção. Alguns desses aspectos, que assumem importância fundamental, são: a composição da solução nutritiva e a vazão de

\footnotetext{
'Engenheiro Agrônomo, Doutor em Produção Vegetal - Departamento de Agronomia/DAG - Universidade Estadual de Maringá/UEM - Avenida Colombo, 5790 - 87020-900 - Maringá, PR - jrhelbel@ibest.com.br

Engenheiro Agrícola, Doutor, Professor Adjunto - Departamento de Agronomia/DAG - Universidade Estadual de Maringá/UEM - Avenida Colombo, 5790 - 87020-900 - Maringá, PR - rrezende@uem.br

${ }^{3}$ Engenheiros Agrícolas, Doutores - Departamento de Agronomia/DAG - Universidade Estadual de Maringá/UEM - Avenida Colombo, 5790 - $87020-900$ Maringá, PR - pslfreitas@uem.br; acagoncalves@uem.br

${ }^{4}$ Engenheiro Agrônomo, Doutor - Departamento de Engenharia Rural - Escola Superior de Agricultura Luiz de Queiroz/ESALQ - Universidade de São Paulo/USP - Avenida Pádua Dias, 11 - Cx. P. 09 - 13418/900 - Piracicaba, SP - frizzone@esalq.usp.br
} 
aplicação dessa solução, que melhor se adeqüe à espécie cultivada e às condições locais de produção. Em se tratando de formulação de soluções nutritivas, já foram divulgadas aproximadamente 300 fórmulas em todo o mundo para as diversas culturas (SCHWARZ, 1995). Andriolo (2002) afirma que a solução nutritiva é o elemento essencial na hidroponia estrita (sem substrato), pois dela depende inteiramente o crescimento da cultura e ela deve conter todos os nutrientes minerais exigidos pelas plantas e também o oxigênio indispensável para a respiração das raízes. Entretanto, não existe uma formulação considerada ideal, pois estão envolvidos um número considerável de variáveis e suas interações (RODRIGUES, 2002).

Em cultivos hidropônicos é usual avaliar o teor de nutrientes na solução nutritiva de forma indireta, medindo sua condutividade elétrica (CE) (VERDONCK et al., 1981). Afinal, a solução nutritiva é composta em grande parte por elementos metálicos e o nível estimado da concentração desses nutrientes pode ser obtido medindo-se a capacidade da solução nutritiva de conduzir corrente elétrica, ou seja, quanto maior a concentração de nutrientes, maior será a capacidade da solução nutritiva de conduzir corrente elétrica (STAFF, 1998). Para alface, a CE da solução nutritiva utilizada geralmente oscila entre 1,6 a 1,8 dS/m (SOARES, 2002) até 2,5 dS/m (CASTELLANE \& ARAÚJO, 1995).

De acordo com Martinez (1997), durante a absorção de nutrientes pelas plantas de alface cultivadas hidroponicamente, ocorre uma diminuição de todos os elementos da solução nutritiva, refletindo, portanto na CE. Existe muita controvérsia com relação ao melhor valor de condutividade elétrica a ser adotado para o cultivo da alface em hidroponia. Acredita-se também que esses valores devem variar de acordo com a cultivar adotada, bem como com as condições climáticas.

Segundo Carmelo (1996) a absorção de nutrientes é um processo que requer energia, que é obtida através do metabolismo da planta, à custa da respiração. Há necessidade portanto, de oxigênio, para que as raízes das plantas possam absorver os elementos essenciais ao seu desenvolvimento. Entretanto, uma solução que esteja com sua superfície em contato direto com a atmosfera, pode não apresentar concentração de oxigênio $\left(\mathrm{O}_{2}\right)$ dissolvido, suficiente para que as raízes de certas espécies respirem, como por exemplo, a alface. A quantidade adicional de oxigênio deve ser fornecida por meio da circulação da solução, quando se faz a irrigação dos canais de cultivo. Conforme o mesmo autor é importante que pesquisas sejam ainda desenvolvidas, principalmente sobre o efeito da taxa de fluxo (vazão), que na maioria dos sistemas em uso, oscila entre 1,5 a 2,0 L.min ${ }^{-1}$, em cada canal.
Assim, diante dessa realidade e considerando que as informações atualmente disponíveis em relação à combinação soluções nutritivas e vazões ainda não são suficientemente esclarecedoras, objetivou-se, no presente trabalho, estudar a produção de alface hidropônica, em função da composição da solução nutritiva e de diferentes vazões.

\section{MATERIAL E MÉTODOS}

O experimento foi realizado no período compreendido entre 31 de julho a 04 de setembro de 2003, em casa de vegetação, na unidade de pesquisa em agricultura irrigada da Universidade Estadual de Maringá (UEM), em Maringá - PR, cujas coordenadas geográficas são: $23^{\circ} 25^{\prime}$ 'de latitude sul, $51^{\circ} 57^{\prime}$ de longitude oeste e com altitude de 542 metros. A casa de vegetação utilizada foi uma estufa do tipo semi-arco com cobertura de polietileno com $150 \mathrm{~mm}$ de espessura, nas seguintes dimensões: 5,0 m de largura; 12,0 m de comprimento; 1,6 $\mathrm{m}$ de altura de pé direito e 2,1 m de altura de vão central.

No interior da estufa, foram construídas três bancadas de cultivo em sistema hidropônico, tipo NFT (Nutriente Film Technique. Cada bancada possuía um desnível de $2 \%$. As bancadas foram constituídas de 6 canais de cultivo com $5 \mathrm{~cm}$ de profundidade, $6 \mathrm{~m}$ de comprimento, propiciando espaçamento de $30 \mathrm{~cm}$ entre plantas nos canais e $22 \mathrm{~cm}$ entre plantas de canais distintos.

No armazenamento das soluções nutritivas testadas utilizaram-se 3 reservatórios, um para cada bancada de cultivo, com capacidade total de 100 litros cada um, porém, trabalhando constantemente com $80 \%$ dessa capacidade. Esses reservatórios foram instalados abaixo do nível das bancadas de cultivo das plantas, permitindo assim, o retorno da solução nutritiva, por meio de uma tubulação de $75 \mathrm{~mm}$ de diâmetro.

Foram medidas diariamente a condutividade elétrica e o pH das soluções testadas. Apesar da variação dos valores de $\mathrm{pH}$ das soluções, esses se mantiveram numa faixa adequada quanto à disponibilidade de nutrientes, e assim não foi necessária nenhuma forma de ajuste.

O sistema de bombeamento da solução nutritiva do reservatório para o início dos canais de cultivo foi composto por uma motobomba de máquina de lavar, com potência de $32 \mathrm{~W}$, instalada de forma afogada e acionada por meio de um temporizador (timer), que iniciava a circulação da solução às 7 horas, sendo o bombeamento intermitente, circulando-se a solução a intervalos de 15 minutos, por períodos de duração de 15 minutos até às 19 horas. Durante a noite, o temporizador acionava o bombeamento durante 15 minutos em intervalos de 2 horas. 
A cultivar de alface utilizada no experimento foi a Vera. De acordo com Della-Vecchia et al. (1999) ela apresenta plantas vigorosas com folhas crespas, eretas e de coloração verde-clara brilhante. Seu ciclo, da semeadura ao ponto ideal de colheita para o mercado, varia de 50 a 70 dias, conforme a região ou época de cultivo. 'Vera' apresenta excelente resistência ao florescimento prematuro em cultivo de verão e apresenta também excelente performance em cultivo de inverno a campo aberto e em cultivo hidropônico, durante o ano todo.

As mudas foram preparadas em bandejas de 200 células de poliestireno expandido, contendo substrato comercial (Plantimax) e transplantadas nas bancadas de cultivo tinham 28 dias de idade, apresentavam de 3 a 4 folhas, cerca de 8 a $10 \mathrm{~cm}$ de altura .

O manejo de reposição adotado foi o de repor diariamente a quantidade de solução nutritiva original absorvida pelas plantas e a água evapotranspirada, acompanhada de uma adição proporcional das mesmas e respectivas soluções nutritivas concentradas que fazem parte dos tratamentos.

Os tratamentos foram constituídos por três soluções nutritivas com diferentes concentrações, expressas por valores de condutividade elétrica (CE) distintas: solução 1 (S1), apresentando $\mathrm{CE}=0,8 \mathrm{dS} / \mathrm{m}$, solução 2 (S2), apresentando $\mathrm{CE}=1,2 \mathrm{dS} / \mathrm{m}$ e solução 3 (S3), apresentando $\mathrm{CE}=2,5 \mathrm{dS} / \mathrm{m}$. Todas combinadas em duas vazões: vazão $1=$ 0,8 L.min ${ }^{-1}$ e vazão $2=1,2 \mathrm{~L} \cdot \mathrm{min}^{-1}$, alternadamente, nos canais das bancadas de cultivo, formando os seguintes tratamentos: $\mathrm{S}_{1} \mathrm{~V}_{1}$ (Solução nutritiva 1 aplicada na vazão 1); $\mathrm{S}_{1} \mathrm{~V}_{2}$ (Solução nutritiva 1 aplicada na vazão 2 ); $\mathrm{S}_{2} \mathrm{~V}_{1}$ (Solução nutritiva 2 aplicada na vazão 1 ); $S_{2} V_{2}$ (Solução nutritiva 2 aplicada na vazão 2); $\mathrm{S}_{3} \mathrm{~V}_{1}$ (Solução nutritiva 3 aplicada na vazão 1); $\mathrm{S}_{3} \mathrm{~V}_{2}$ (Solução nutritiva 3 aplicada na vazão 2 ).
A opção pelas soluções 1 e 2 teve como critério a escolha de soluções nutritivas que, de forma geral, são empregadas tanto na pesquisa científica, como também divulgadas na literatura técnica disponível aos profissionais das ciências agrárias e aos próprios produtores e empresários agrícolas. A escolha da solução 3 teve como critério optar por uma solução com maior concentração de nutrientes em comparação com as soluções 1 e 2 , encontradas na literatura científica. Partindo desse propósito, as soluções 1, 2 e 3 foram preparadas de acordo com as recomendações de seus autores, respectivamente: Carmelo (1996), Furlani et al. (1999) e Sasaki (1992). As concentrações dos nutrientes podem ser verificadas nas Tabelas 1 e 2 .

Para preparar cada solução utilizaram-se como fontes dos nutrientes sais fertilizantes, em detrimento de formulações comerciais já prontas existentes no comércio. Na Tabela 3, podem ser observados os sais empregados em cada solução e suas respectivas quantidades.

Durante o período experimental, foram registradas, diariamente, com o auxílio de um termômetro, as temperaturas máxima, média e mínima do ar no interior da casa de vegetação, na altura das bancadas de cultivo. Os valores podem ser observados na Figura 1.

As plantas foram colhidas quando alcançaram o ponto de colheita, o qual ocorreu 35 dias após o transplante das mudas. Foram avaliadas as variáveis biomassa fresca da parte aérea e diâmetro do caule, por meio de balança e de paquímetro digitais.

$\mathrm{O}$ delineamento experimental utilizado foi inteiramente casualizado, no esquema fatorial 3 x 2, composto por três soluções nutritivas e 2 vazões de aplicação, com 3 repetições, sendo cada repetição composta por 20 plantas. Cada parcela experimental foi constituída por uma linha de cultivo e cada bancada abrigou 6 parcelas.

Tabela 1 - Concentrações de macronutrientes da solução básica (mg/L) para o cultivo hidropônico de alface.

\begin{tabular}{cccccccc}
\hline & $\mathrm{N}_{-N O}{ }^{-}$ & $\mathrm{N}^{-} \mathrm{NH}_{4}{ }^{+}$ & $\mathrm{P}$ & $\mathrm{K}$ & $\mathrm{Ca}$ & $\mathrm{Mg}$ & $\mathrm{S}$ \\
\hline Sol. 1 & 86,8 & 7,9 & 10,9 & 155 & 58 & 12 & 16 \\
Sol. 2 & 174 & 24 & 32,7 & 193 & 183 & 39,4 & 52 \\
Sol. 3 & 208 & 28,5 & 32,7 & 232 & 292,8 & 49,3 & 66 \\
\hline
\end{tabular}

Tabela 2 - Concentrações de micronutrientes da solução básica (mg/L) para o cultivo hidropônico de alface.

\begin{tabular}{ccccccc}
\hline & $\mathrm{B}$ & $\mathrm{Cu}$ & $\mathrm{Fe}$ & $\mathrm{Mn}$ & $\mathrm{Mo}$ & $\mathrm{Zn}$ \\
\hline Sol. 1 & 0,2 & 0,02 & 3,6 & 0,2 & 0,007 & 0,02 \\
Sol. 2 & 0,3 & 0,04 & 3,6 & 0,3 & 0,08 & 0,11 \\
Sol. 3 & 0,5 & 0,01 & 3,6 & 0,4 & 0,07 & 0,02 \\
\hline
\end{tabular}


Tabela 3 - Quantidade de Sais/Fertilizantes, em mg/L, utilizados para compor as soluções nutritivas dos tratamentos.

\begin{tabular}{lccc}
\multicolumn{1}{c}{ Fertilizante } & $\begin{array}{c}\text { Solução 1 } \\
\text { mg/L }\end{array}$ & $\begin{array}{c}\text { Solução 2 } \\
\text { mg/L }\end{array}$ & $\begin{array}{c}\text { Solução } 3 \\
\text { mg/L }\end{array}$ \\
\hline Nitrato de cálcio & 240 & 750 & 1200 \\
Nitrato de Potássio & 400 & 500 & 260 \\
Fosfato monoamônio(MAP) & 50 & 150 & 150 \\
Sulfato de magnésio & 120 & 400 & 500 \\
Cloreto de potássio & - & - & 250 \\
Sulfato de Manganês & 1,0 & 1,50 & 1,81 \\
Ácido Bórico & 1,15 & 1,50 & 2,86 \\
Sulfato de Zinco & 0,09 & 0,50 & 0,10 \\
Sulfato de Cobre & 0,04 & 0,15 & 0,04 \\
Molibdato de sódio & 0,013 & 0,15 & 0,15 \\
Fe-EDTA & 40 & 40 & 40 \\
\hline
\end{tabular}

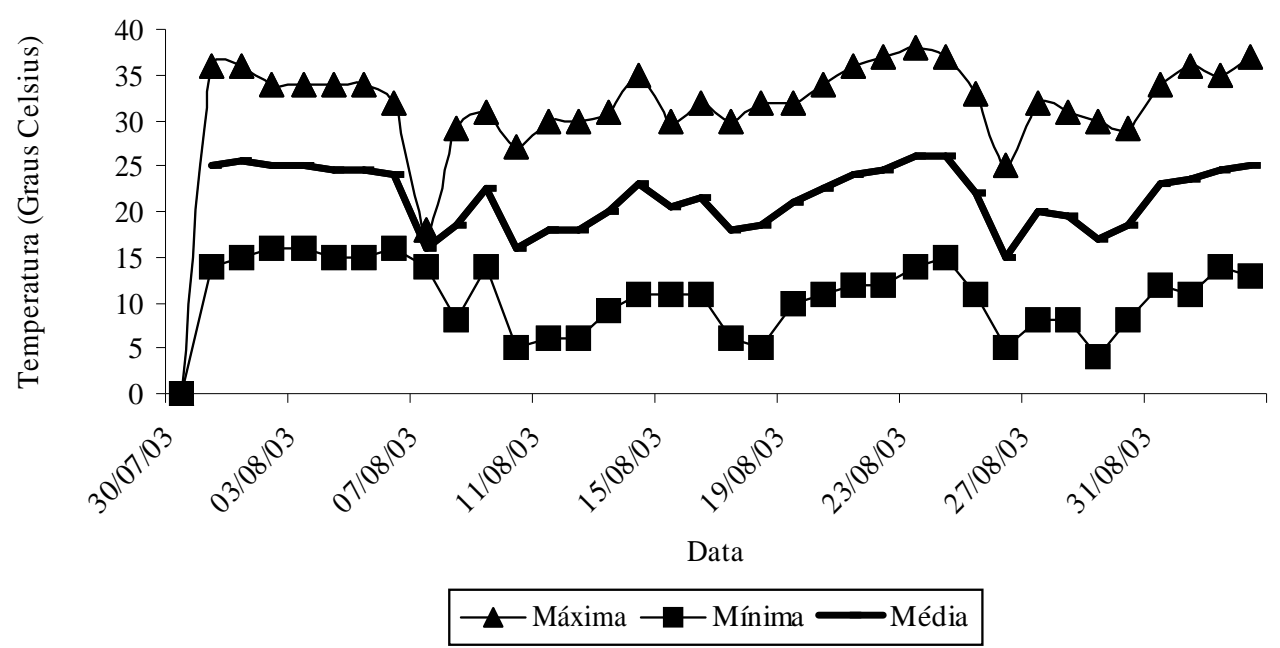

Figura 1 - Valores de temperaturas máxima, média e mínima medidos durante o período experimental. Maringá, UEM, 2003 .

\section{RESULTADOS E DISCUSSÃO}

Não houve interação significativa entre os fatores solução e vazão, indicando que atuam independentemente. Também não houve efeito significativo para o fator vazão quando estudado isoladamente, indicando que as vazões de 0,8 e 1,2 L.min ${ }^{-1}$, não influenciaram a produção das plantas. Possivelmente, esses valores de vazões testados, não contribuíram para que, junto com outros fatores, houvesse uma melhor oxigenação das soluções, beneficiando a respiração das raízes e, conseqüentemente, a absorção de nutrientes.
A Tabela 4 demonstra que a $\mathrm{S} 2$ teve um efeito superior e significativo na biomassa fresca e no diâmetro de caule das plantas. Comparando os resultados dessa solução, em relação à S3, que apresenta a maior condutividade elétrica (CE), as diferenças observadas provavelmente estão relacionadas a efeitos negativos ocasionados pela pressão osmótica (Tabela 4). Pois, de acordo com Taiz \& Zaiger (2002), o aumento da concentração salina aumenta a pressão osmótica no meio fazendo com que, mesmo em um ambiente com água disponível como é a solução nutritiva, a planta tenha 
Tabela 4 - Variação da condutividade elétrica, pressão osmótica, biomassa fresca e diâmetro do caule das plantas de alface em função da aplicação de diferentes composições da solução nutritiva. Maringá, UEM, 2003.

\begin{tabular}{ccccc}
\hline $\begin{array}{c}\text { Soluções } \\
\text { Nutritivas }\end{array}$ & $\begin{array}{c}\text { Condutividade } \\
\text { elétrica - CE }\end{array}$ & $\begin{array}{c}\text { Pressão } \\
\text { Osmótica* }\end{array}$ & $\begin{array}{c}\text { Biomassa } \\
\text { fresca }\end{array}$ & $\begin{array}{c}\text { Diâmetro } \\
\text { do caule }\end{array}$ \\
\hline S1 & $-----d S / m----$ & $------a t m-----$ & ------- g-------- & $--------m m-------$ \\
S2 & 0,8 & 0,22 & $267,9 \mathrm{~b}$ & $23,3 \mathrm{~b}$ \\
$\mathrm{~S} 3$ & 1,2 & 0,34 & $413,4 \mathrm{a}$ & $26,0 \mathrm{a}$ \\
$\mathrm{CV} \%$ & 2,5 & 0,70 & $310,9 \mathrm{~b}$ & $21,7 \mathrm{~b}$ \\
\hline
\end{tabular}

Médias seguidas pela mesma letra, na coluna, não diferem entre si, em nível de 5\% de probabilidade, pelo teste de Tukey.

*Calculada a partir dos valores de condutividade elétrica das soluções nutritivas, segundo Carmelo (1996), através da equação P.O $=0,28 \times \mathrm{CE}$.

dificuldade em absorver água suficiente para repor a perda por transpiração.

Considerando que a cultura da alface em temperaturas acima de $25^{\circ} \mathrm{C}$ acelera seu ciclo, resultando em plantas menores (FILGUEIRA, 1982) e que, segundo Furlani et al. (1999), em regiões de clima tropical é conveniente trabalhar com soluções mais diluídas (menor $\mathrm{CE}$ ), a variação da temperatura média durante o período experimental (Figura 1), pode também ter contribuído para o menor desenvolvimento vegetativo das plantas cultivadas na S3.

Os resultados observados para $\mathrm{S} 2(1,2 \mathrm{dS} / \mathrm{m})$, comparada com S1 (0,8 dS/m), podem ser conseqüência da carência de nutrientes minerais na sua composição, verificada através de sua menor $\mathrm{CE}$, sugerindo que apenas a disponibilidade dos elementos minerais na rizosfera, pode não ser suficiente para um crescimento vegetal satisfatório; mas é preciso que os nutrientes estejam nas concentrações e relações adequadas, principalmente no cultivo hidropônico, onde a deficiência ou a toxidez de um determinado nutriente mineral se manifesta mais rapidamente do que no solo, pois a inércia química do solo é maior do que na solução nutritiva. Conforme Adams (1994) e Furlani et al. (1999), em se tratando de cultivos hidropônicos, a absorção de água e nutrientes pelas plantas é proporcional à concentração de nutrientes na solução próxima às raízes.

Huett (1994), cultivando plantas de alface (cv. Coolguard), em solução com baixa CE ( $0,4 \mathrm{dS} / \mathrm{m})$, observou deficiências de nitrogênio e potássio, e altos teores de cálcio em folhas novas, sendo que as deficiências diminuíram com o aumento da $\mathrm{CE}$ da solução nutritiva. Embora não tenham sido apresentados dados relativos a análises químicas de folhas, a baixa absorção de nitrogênio e potássio pode ter sido a causa da menor produção de biomassa fresca na menor condutividade.

A produção de biomassa fresca por planta de alface obtida com S2 (413,4 g por planta) merece destaque e mostrou-se superior aos encontrados por Koefender (1996), Shmidt et al. (2001) e Vaz \& Junqueira (1998) que, produzindo alface (cv. Verônica) em sistema NFT, obtiveram, respectivamente, médias de 207,8 g, 183,4 g e 295,8 g de biomassa fresca, por planta.

Confrontando os resultados para diâmetro de caule, apresentados pela $\mathrm{S} 2$ (26,0 $\mathrm{mm})$, com $\mathrm{S} 1$ (23,3 mm) e S3 (21,7 mm), observa-se um provável efeito da CE das respectivas soluções influenciando na vascularização das plantas. A maior concentração de nutrientes (S3) pode ter restringido a absorção de água, reduzindo a biomassa fresca e isso repercutiu num menor desenvolvimento do sistema vascular. Enquanto a menor concentração (S1) pode ter sido nutricionalmente insuficiente, principalmente em nitrogênio na forma nítrica. Pois esse nutriente desempenha papel importante, como soluto osmoticamente ativo, no processo de alongamento celular, que influencia a vascularização das plantas e, conseqüentemente, a biomassa fresca.

\section{CONCLUSÕES}

Apenas a condutividade elétrica da solução nutritiva influenciou a biomassa fresca e o diâmetro de caule da alface hidropônica, sendo os maiores valores obtidos com a CE de 1,2 dS/m.

\section{REFERÊNCIAS BIBLIOGRÁFICAS}

ADAMS, P. Nutrition of greenhouse vegetables in NFT na hydroponic systems. Acta Horticulturae, The Hague, n. 361, p. 254-257, 1994. 
ALBERONI, R. B. Hidroponia: como instalar e manejar o plantio de hortaliças dispensando o uso do solo. São Paulo: Nobel, 1998. 102 p.

ANDRIOLO, J. L. Olericultura geral. Santa Maria: UFSM, 2002. 158 p.

CARMELLO, Q. A. C. Cultivo hidropônico de plantas. Piracicaba: ESALQ-USP, 1996. 43 p.

CASTEllane, P. D.; ARAÚJO, J. A. de. Cultivo sem solohidroponia. 4. ed. Jaboticabal: Funep, 1995. 43 p.

DELLA-VECCHIA, P. T.; KOCH, P. S.; KIKUCHI, M. Vera: nova cultivar de alface crespa resistente ao florescimento prematuro. Horticultura Brasileira, Brasília, v. 17, n. 2, p. 171, jul. 1999.

FILGUEIRA, F. A. R. Manual de olericultura: cultura e comercialização de hortaliças. 2. ed. São Paulo: Agronômica Ceres, 1982. v. 2, 587 p.

FURLANI, P. R.; SILVEIRA, L. C. P.; BOLONHEZI, D.; FAQUIN, V. Cultivo hidropônico de plantas. Campinas: IAC, 1999. 52 p. (Boletim técnico, 180).

HUETT, D. O. Growth, nutrient uptake and tipburn sevety of hidroponic lettuce in response to electrical conductivity and $\mathrm{K}: \mathrm{Ca}$ ratio in solution. Australian Journal of Agricultural Research, Victoria, v. 45, p. 251-267, 1994.

KOEFENDER, V. N. Crescimento e absorção de nutrientes pela alface cultivada em fluxo laminar de solução. 1996. 85 f. Dissertação (Mestrado) - Escola Superior de Agricultura “Luiz de Queiroz”, Universidade de São Paulo, Piracicaba, 1996.

MARTINEZ, H. E. P. Formulação de soluções nutritivas para cultivos hidropônicos comerciais. Jaboticabal: Funep, 1997. $31 \mathrm{p}$.
RODRIGUES, L. R. F. Técnicas de cultivo hidropônico e de controle ambiental no manejo de pragas, doenças e nutrição vegetal em ambiente protegido. Jaboticabal: Funep/Unesp, 2002. 762 p.

SASAKI, J. L. S. Hidroponia. In: SEMANA DA AGRONOMIA, 11., 1992, Ilha Solteira. Palestras... Ilha Solteira: Unesp, 1992.9 p.

SCHMIDT, D.; SANTOS, O. S.; BONNECARRÉRE, R. A. G.; MARIANI, O. A.; MANFRON, P. A. Desempenho de soluções nutritivas e cultivares de alface em hidroponia. Horticultura Brasileira, Brasília, v. 19, n. 2, p. 122-126, jul. 2001.

SCHWARZ, M. Soilles culture management. [S.1.]: Springer-Verlag, 1995. $195 \mathrm{p}$.

SOARES, I. Alface: cultivo hidropônico. Fortaleza: UFC, 2002.

STAFF, H. Hidroponia. 2. ed. Cuiabá: Sebrae/MT, 1998. $86 \mathrm{p}$.

TAIZ, L.; ZAIZER, E. Plant physiology. Redwood: The Benjamin, 2002. 559 p.

VAZ, R. M. R.; JUNQUEIRA, A. M. R. Desempenho de três cultivares de alface sob cultivo hidropônico. Horticultura Brasileira, Brasília, v. 16, n. 2, p. 178-189, nov. 1998.

VERDONCK, O.; VLEESCHAUWER, D.; BOODT, M. The influence of the substrate to plant growth. Acta Horticulturae, Wageningen, v. 126, p. 251-258, 1981.

ZITO, R. K.; FRONZA, V.; MARTINEZ, H. E. P.; PEREIRA, P. R. G.; FONTES, P. C. R. Fontes de nutrientes, relações nitrato: amônio e molibdênio, em alface (Lactuca sativa L.) produzida em meio hidropônico. Revista Ceres, Viçosa, v. 41, n. 236, p. 419-430, 1994. 